\title{
In-situ ETEM Studies of Fe Catalyst NPs Formation under Molecular or Radicals/Activated Hydrogen Environments for the Growth of SWCNTs
}

$\underline{\text { Ileana Florea }}{ }^{1}$, Mariam Ezzedine ${ }^{1}$, Mihai-Robert Zamfir $^{1,2}$, Loan Truong ${ }^{1}$, Eleonor Caristan ${ }^{1}$ and Costel - Sorin Cojocaru ${ }^{1}$

${ }^{1}$ LPICM, CNRS, Ecole polytechnique, IPParis, 91228 Palaiseau, France.

${ }^{2}$ National Institute for Laser, Plasma \& Radiation Physics (INFLPR), Atomistilor Street, No. 409, Magurele, Ilfov RO-077125, Romania.

Catalyst design plays vital roles in the structurally relevant chemical reactions. Revealing the catalyst structure and associated chemical activity in the reactive environment and at the atomic scale is imperative for the rational design of catalysts as well as for the investigation of reaction mechanism [1,2]. However in situ characterization at the atomic scale at high temperature is still a great challenge. [3]-[5]. The present study is focused on the realization of in situ TEM observations of Fe catalyst film dewetting and catalytic nanoparticles (NPs) formation under molecular and/or radical/ activated hydrogen environments. The formed NPs exhibit different sizes depending on the temperature and type of reactive gas. The experiments are performed within a modified environmental transmission electron microscope-ETEM equipped with a Cs image aberration-corrector; a special sample holder and customized CVD gas sources that allow sending collimated beams of molecules/radicals with different partial pressure.

From the experimental point of view the analysis of the recorded images at different temperatures and exposure times allowed us observing that the Fe film dewetting is strongly related to both the nature of hydrogen environment and the temperature conditions. Thus, through in-situ TEM heating process under $3 \mathrm{sccm}\left(1.410^{-4} \mathrm{mbar}\right)$ of molecular $\mathrm{H} 2$ environment we notice that the Fe film dewetting starts at $400^{\circ} \mathrm{C}$ leading to the formation of big crystalline Fe nanoparticle (see Figure1). We have observed that the Fe NPs size increases, 20nm size, as the temperature increases while keeping the same H2 flow. On the contrary, under $6 \mathrm{sccm}\left(2.510-{ }^{3}\right.$ mbar $)$ activated hydrogen environment the Fe film dewetting initiate only at higher temperature, around $500^{\circ} \mathrm{C}$ with the formation of smaller crystalline $\mathrm{Fe} \mathrm{Nps}$ with sizes ranging from $1 \mathrm{~nm}$ up to $5 \mathrm{~nm}$ (see Figure2). The formation of such small nanoparticle is related to the presence of hydrogen radicals which tend to block the metal atoms surface diffusion by creating small defects within the support which pin the forming nanoparticle and prevent their agglomeration.

\section{References:}

[1] MC Hersam et al, Nat. Nanotechnol. 3 (2008), p. 387.

[2] AR Harutyunyan et al, Science 326 (2009), p. 116.

[3] S Hofmann et al, Nano Lett. 7 (2007), p. 602.

[4] R Sharma et al, J. Mater. Res. 20 (2005), p. 1695.

[5] M Picher et al, Nano Lett. 14, 6104 (2014).

[6] We acknowledge the ANR for funding the NanoMAX ETEM through the TEMPOS grant for "Equipements d'excellence", project number 10-EQPX-0050 and GIANT project. Thanks are also due to the CIMEX for the use of the electron microscopes at École polytechnique. 

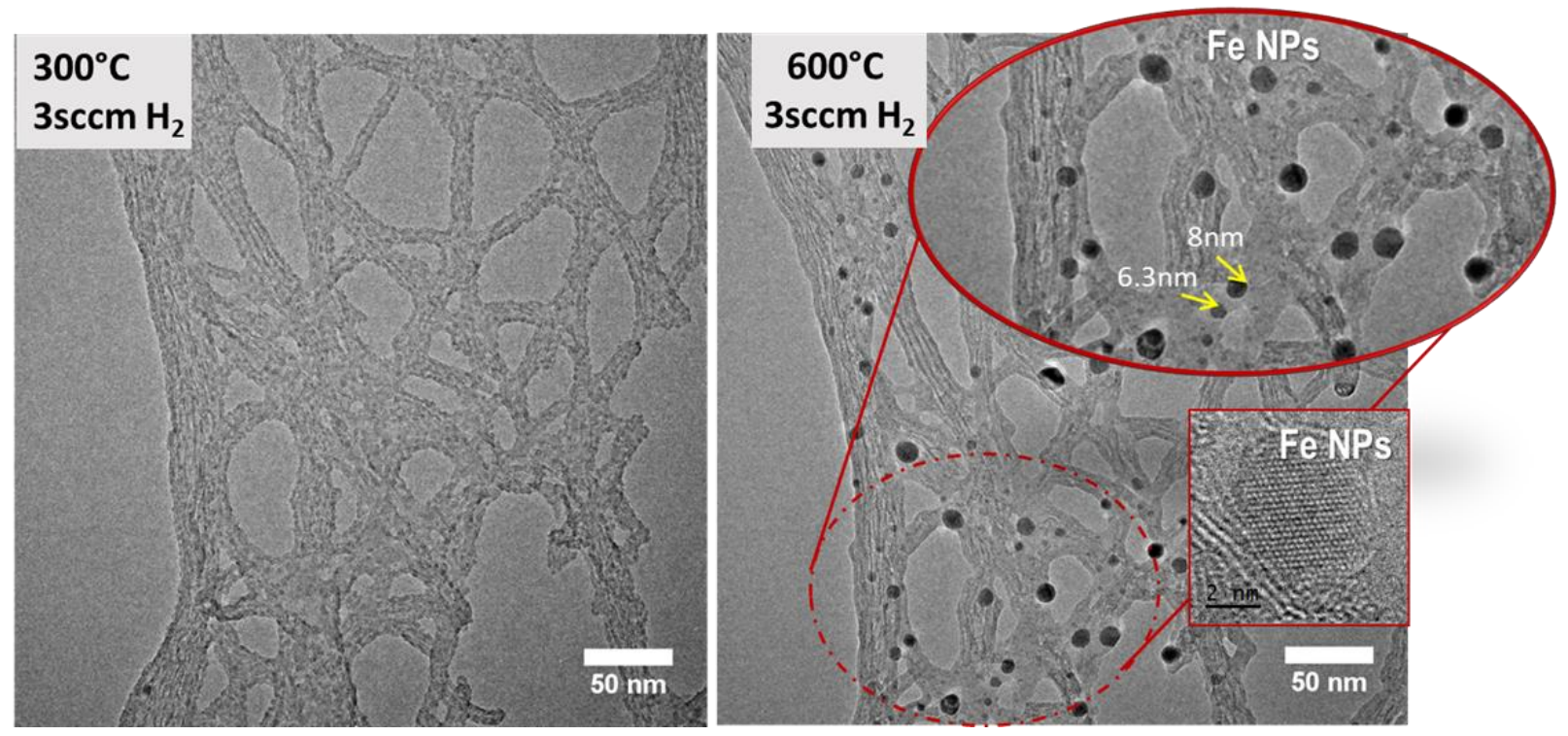

Figure 1. In-situ TEM analysis of Fe film dewetting through heating process under $3 \mathrm{sccm}$ molecular $\mathrm{H} 2$ environment illustrating the formation of Fe NPs.
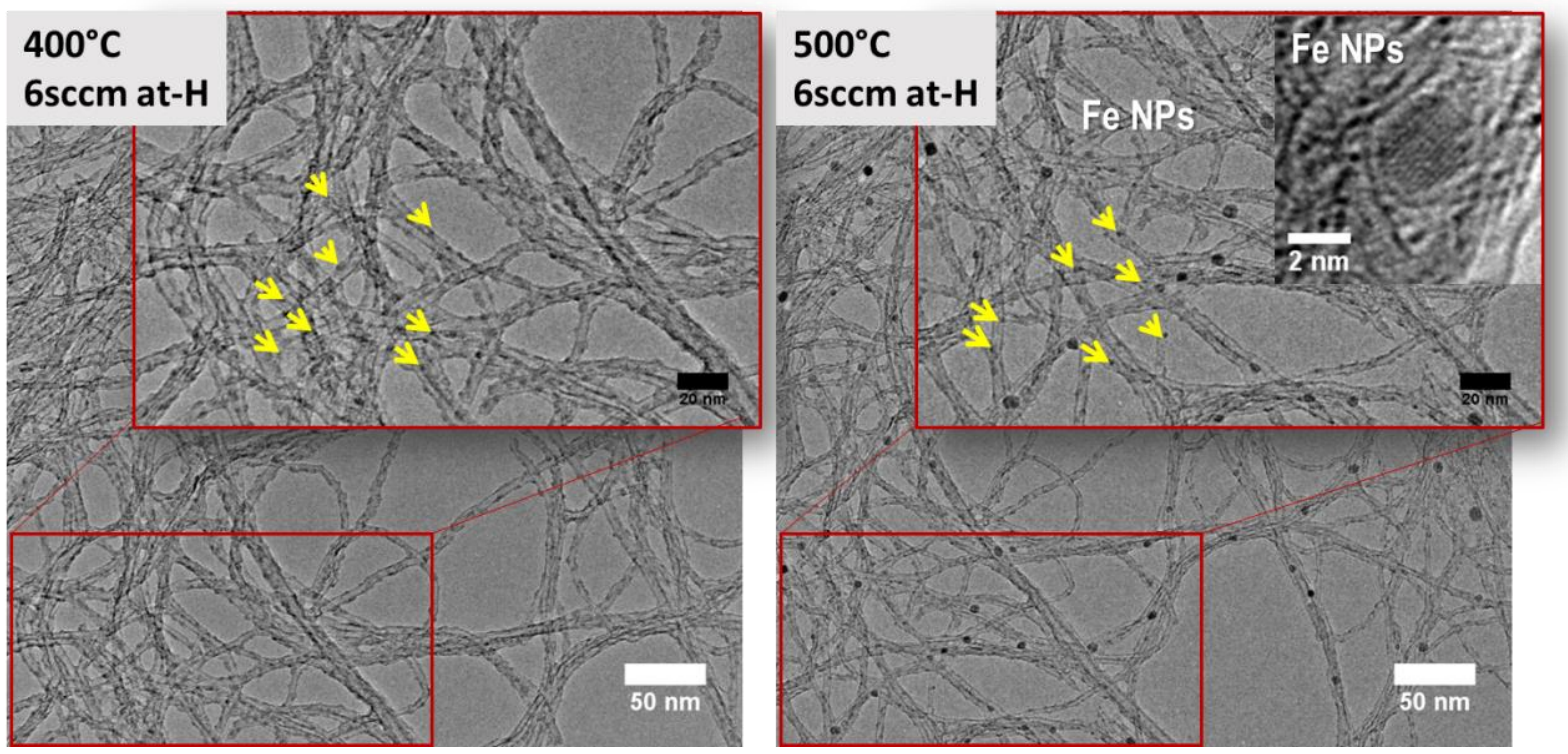

Figure 2. In-situ TEM analysis of Fe film dewetting through heating process under 6 sccm activated $\mathrm{H} 2$ environment illustrating the formation of small Fe metallic NPs. 